\title{
Disaggregate GIS modelling to track spatial change: exploring a decade of commuting in South East Queensland, Australia
}

\author{
Authors: Tiebei $\mathrm{Li}^{\mathrm{i}}$, Jonathan Corcoran ${ }^{\mathrm{ii}}$, Matthew Burke ${ }^{\mathrm{i}}$ \\ Tiebei $\mathrm{Li}^{\mathrm{i}^{*}}$ \\ Urban Research Program, Griffith University, 170 Kessels Road, Nathan, Queensland, 4111, Australia. \\ Email for correspondence: t.li@griffith.edu.au Phone: +61-7-33656716. \\ Jonathan Corcoran ${ }^{i i}$ \\ School of Geography, Planning and Environmental Management, The University of Queensland, Brisbane QLD \\ 4072 Australia \\ Matthew Burke ${ }^{i}$ \\ Urban Research Program, Griffith University, 170 Kessels Road, Nathan, Queensland, 4111, Australia.
}

\begin{abstract}
Changes in commuting dynamics are explored for a large geographical region using journeyto-work (JTW) data derived from two censuses (1996 and 2006). The main thrust is concerned with the identification of geographic patterning of commuting distance and flow (i.e. the number of people travelling from region $i$ to region $j$ ) coupled with the changes in these patterns over the decade to 2006. GIS-based methods are employed to first calculate the average commuting distance and second to map the degree of self-containment (i.e. people living and working within the same area) at a fine spatial scale. Through a quantitative analysis of JTW patterns over the two census data sets, the results are linked to current planning debates regarding urban spatial policies that seek to reduce commuting distances. Specific attention is given to address: (1) spatial variation in changes in commuting distance across the study region between 1996 and 2006; and (2) whether jobs-housing balanced development reduced commuting distances over the decade. Results indicate that the spatial patterns of commuting have changed little over the 10 year period and the change in commuting distance presented strong relationships to the change in jobs-housing ratio (JHR) at local areas. Implications for urban policy include the need for improved industry-occupation matching and consideration of planned employment decentralisation.
\end{abstract}

Key words: journey-to-work, jobs-housing ratio, Geographical Information Systems, exploratory spatial data analysis 


\section{Introduction}

Commuting constitutes a typical daily activity for most workers, placing significant demands on our transportation systems, and is linked to their sub-optimal performance (Horner, 2004). As cities grow and urban structure changes, the spatial dynamics and volume of commuter flows have significant implications for transport and land use planners. Congestion has become a global reality in urbanised areas and urban structure is seen as a 'solution', amongst many, for managing travel demand (Rickwood and Glazebrook, 2007). At the same time, employment decentralization has been occurring in many Western cities, much of it unplanned and marketled, changing commuting patterns. Along with increasing challenge for urban transport, key transport issues are also affecting workers in the rural and regional areas including lower access to employment, longer commutes to work and increased transport energy cost. Such transport issues in the rural commuting are often overlooked by journey-to-work (JTW) studies. Developing an understanding of the broad relationship between a worker's residence to their locale of work and commuting behaviour in a given region, its variation over space and time and how this differs according to industry sector and travel mode are central to the search for solutions to transport problems.

Tracking changes in the spatial dimension of commuter behaviour can help identify the ever varying nature of travel demand. Whilst there has been a great deal of attention that has focused on the spatial nature of urban form and commuting (Cervero, 1989; Wachs et al., 1993; Giuliano and Small, 1993; Gipps et al, 1997) there has been very little research that has investigated spatial dynamics of commuting and its variation over time at a spatially disaggregated scale. Numerous studies have examined how JTW changes are associated with change in jobs-housing relationship in the metropolitan areas to identify the forces of jobshousing relationship that may be influencing JTW patterns. There is relatively little research that has explored commuting changes for all workers, not just those within metropolitan areas but also who reside in rural and regional areas and their association with changes in jobs and housing structure at the large geographical level. Building on the work of Horner (2007), this research uses methods to explore the spatial dynamics of commuting for a large geographical region using spatially disaggregate JTW data for two census periods (1996 and 2006). This work is applied to investigate the geographic pattern of commuting distance and jobs-housing relationship across South East Queensland (SEQ), the fastest growing region in Australia. As will be shown, SEQ provides an interesting case for exploring commuting flows, having quite a different urban/regional structure to US cities, and also being a multi-city region with a large peri-urban area. Specific attention is given to address two questions: (1) spatial variation in changes in commuting distance across the large region between 1996 and 2006; and (2) whether perceived jobs-housing balance (JHB) reduced commuting distances over the decade. JHB may be defined as when the ratio of jobs to residents in the workforce within a given area approaches 1:1. A number of spatial techniques are used to analyse the commuting data, including advances in some methodological advances in the statistical analyses one can use to explore such changes. In order to identify the spatial variation of JHB forces that may influence JTW patterns across the region, a local spatial statistical method are used. The main contributions of this paper are mainly in the application to the SEQ case, however the paper also provides advances on methods established by others (Horner 2007; Wang 2000) and new methods for investigating these issues with local spatial statistics. In so-doing we aim to gain a broader perspective on land use-transportation relationships, and to establish if the methodologies and findings are transferable to a different geographical, environmental and social context and comment on any differences in data and analytical approaches that can account for such trends. 
The paper is structured as follows: The following section comprises a review of geographical studies of commuting, further highlighting the research gap. The study area and data is then described in Section 3. Section 4 describes the methods used to model travel distance and JHB using JTW data. The results are then presented in Section 5, examining the effect of JHB on commuting distance in the SEQ region. Finally, Section 6 summarises the major findings, highlighting key limitations before outlining available avenues for future research.

\section{Geographical studies of JTW}

The geographical dimension of urban commuting has received significant attention over time (see for example Mogridge, 1979; O’Connor, 1978, 1980; Gipps et al, 1997; Sultana, 2002; Horner and Murray, 2003; O’Kelly and Lee, 2005; Titheridge and Hall, 2006; Sakanishi, 2006; Weber and Sultana, 2007; Mees et al, 2008). In a comprehensive review, Horner (2004) categorises the range of geographical studies into areas focussing on urban commuting and sustainability, urban commuting and land use, which he sub-divides into studies investigating JHB, excess commuting and accessibility. Examples of studies on commuting dynamics and JHB include Cervero and Wu's (1998) work on commuting in San Francisco, which found that car use and commuting distances increased with employment decentralization, Wang (2000) who found that changes in commuting distance did not appear to be correlated with JHB as well as studies by Wachs et al. (1993) and Peng (1997).

A number of studies have used geographical approaches to focus on spatial changes in commuting over time. Mogridge (1979) investigated changing spatial patterns in JTW in central London between 1966 and 1971, showing an increase in commuting lengths over the period. O’Connor (1978) explored changing commuting patterns in the metropolitan area of Melbourne, Australia, from 1966 to 1971; drawing the conclusion that suburbanization extended the commuting distance from a local to a regional scale. Wachs et al. (1993) focused on a single employer (Kaiser Permanente, based in Southern California) and tracked changes in the home and work locations for a total of 1,500 employees over a 6 year period. They found no significant changes in trip length, despite growth in the local work force contributing to an increase in local traffic congestion. Christopher et al., (1995) used US household travel surveys to explore changes in the direction of travel for 9 counties in the Chicago area between 1970 and 1990. Results indicated that directional biases (for each of the 9 counties) have changed little over the 20 year study period despite increases in car ownership and significant decentralization. Vandersmissen et al. (2003) analysed changes in urban form and commuting time for Québec City between 1977 and 1996. Using socially disaggregate travel survey data they found gender effects in that despite significant decentralization in Québec City's jobs and housing, commuting distances have increased for males and decreased for females. Titheridge and Hall (2006) analysed changes in JTW trips for two transport corridor areas in South East England suggesting that new growth centres may increase car use but not necessarily commute distances. Nielsen and Hovgesen (2008) used the origin-destination commute data from the 1991 and 2001 Census to explore the patterns of commuting flows in England and Wales. They found commuting flows widened and stretched out along a national corridor from London to Manchester between 1991 and 2001 as the result of the decentralization of population and jobs over time. Horner (2007) investigated urban form and commuting change in the metropolitan area of Tallahasse, Florida between 1990 and 2000. Using a pairwise correlation analysis of land use and commuting, the results suggest that urban structure was relatively stable over the 10 year study period, with a strong continuing relationship between land use and commuting patterns. Yates et al. (2006) used more detailed labour market disaggregation to explore JHB in Sydney, SEQ and Melbourne, including by industry/occupation for four selected professions 
(computing professionals, nursing professionals, cleaners and hospitality workers). They found very strong job concentrations in the central city cores (including Brisbane's centre) and especially for the computer professionals for who job deficits in the suburbs were high. However jobs were more dispersed for the other three professions, with job deficits for cleaners and hospitality workers lower in the suburban areas. Their level of spatial aggregation (large local government areas), however, limited the capacity to assess commuter flows within whole cities, such as the Gold Coast.

The studies noted above demonstrate that geographical approaches can be applied to explore changes in commuting trends over time. However, these studies often address the relationship between commuting and urban structure based on limited geographical areas (e.g. small to midsize metropolitan areas), excluding commuting origins and destinations in rural and regional locations. Exploring commuting change for a larger geographical area is important for gaining a broad perspective on transport/land-use relationships to help in assessing structural changes and for subsequent transport and land use plan formulation. Many of these studies have also used higher levels of aggregation in census and travel survey data, which may mask patterns revealed only at finer grained levels of spatial disaggregation. This study aims to address these issues.

\section{The study area and data}

SEQ is the fastest-growing region in Australia, attracting on average 55,000 new residents each year. SEQ has experienced steady employment growth in the last two decades and is now emerging as a significant economic region in Australia (Office of Urban Management, 2005). Geographically, SEQ is a sprawling low density region covering a relatively large geographical area (2.3 million hectares). It comprises three major urban centres. Figure 1 depicts the major urban settlements of SEQ, which are the city of Brisbane and the nearby coastal cities of the Sunshine Coast and Gold Coast. This is a large poly-nuclear region with a very strong employment core in the Brisbane central business district and its immediate frame (upon which the region's public transport network is focused, including over $200 \mathrm{~km}$ of commuter rail lines) (Spearritt, 2009). The population is mainly clustered on the coast with the exception of two regional centres at Ipswich and Toowoomba. The remaining parts of the region are mainly dominated by rural land. Due to its poly-centric structure, the region has developed a complex pattern of JTW movements and economic interactions (Office of Economic and Statistical Research, 2006; Yates et al, p57).

\section{insert Figure 1 here}

Figure 1. Study area

Given the levels of population growth, a significant planning problem is anticipating transport needs in SEQ. In 2005, the Queensland Government passed legislation to implement a new regional growth management plan for the SEQ region (Office of Urban Management, 2005). The plan supports urban consolidation and seeks to achieve better JHB and a more selfcontained structure to support help manage travel demand, processes that were seen as already underway. As such, SEQ's recent experiences in regional restructuring, and the availability of critical datasets, make it a useful location for exploring the nature of spatial changes in commuting. Did perceived improvements in the region's JHB reduce the average commuting distance between 1996 and 2006? 
In this study, the spatial structure of SEQ is more varied than the study areas used for previous research undertaken by Horner (2007) and Vandersmissen et al.(2003) who used Tallahasse (U.S.) and Québec City (Canada) respectively. For example, Tallahasse is a relatively small metropolitan city with a mono-centric urban structure. By contrast, SEQ is a large and polycentric region which exhibits a greater degree of heterogeneity in its urban and regional setting. The region contains a greater number of populations and distinctive mix of economic activities and has developed more complex and diverse geographic patterns in population and employment. Therefore, this study context permits a more rigorous spatial assessment of a broad impact of land use change on JTW patterns at a large regional level.

Australian Bureau of Statistics (ABS) Journey to Work data from the 1996 and 2006 Census of Population and Housing was used in this study. There are two types of information contained in the JTW data sets: GIS layers for both the origin and destination zones for all trips, and the JTW origin-destination matrix referencing the number of trips between each origin and destination. ${ }^{1}$ The advantage of using the ABS JTW data is that it is a complete enumeration of the population, which provides more accurate information on travel demand than that was provided by the sampled travel survey data. It permits a more rigorous analysis of the change in JTW patterns at a spatially disaggregate scale. Its limitation is that it does not allow social disaggregation to industry/occupation type.

\section{Methodology}

We propose a tripartite methodology that collectively has the capacity to explore spatial dynamics of JTW over time at the spatially disaggregated scale. This approach builds in part on the methods and approaches used by Wang (2000) and Horner (2007).

\subsection{Spatial integration of JTW data sets}

A major challenge in the spatial analysis of census data are changes in geographical boundary of traffic zones over time. This creates issues for how independent zonal structures for different years can be spatially integrated into a single, consistent set of geographical units. To overcome this problem, an area interpolation technique was applied to transform the spatial data from the zones supplied by the ABS to a new set of consistent spatial units. We use Statistical Local Area (SLA) as new destination zones for 1996 and 2006. An areal weighted proportioning method (Langford, 2006) assumes homogenous spatial data (in this case - the number of JTW trips) for previous destination zones. The data estimation for the new destination zones was based on the degree of spatial overlap with known data for the previous destination zones. Error evaluation was conducted by examining the consistency between the estimated values and the sum of their constitute destination zone values in the original dataset. Appling the evaluation procedure to the output datasets for 1996 and 2006, the results reported a low aggregation error, smaller than $1 \%$ across the data sets.

\subsection{Commuting distance}

In this section, average commuting distance was computed for each SLA using the road network data to determine the travel distance between each origin and destination. Because

\footnotetext{
${ }^{1}$ The JTW matrix contains 300 origin zones. However, each year contained a different number of destination zones. For example, there were 558 destination zones for the year 1996 and 627 destination zones for the year 2006, for the same region. The reason for this is that the spatial configuration (e.g. shape and size) of destination zones were independently defined for each census year by the Queensland Department of Transport based on the flow of people.
} 
SLAs are a relatively large geographical unit (especially SLAs for rural areas), the measure of JTW distance using geometric centroids in many cases was deemed not to represent the multiple available route possibilities for commuters. To remedy this situation, 10 points were located randomly within each SLA and each point used as the single departing location and arrival location of travel ${ }^{2}$. Average point-to-point network travel distances were computed for each SLA ${ }^{3}$. Each SLA-SLA commuting distance was multiplied by the number of commuting trips between each origin-destination pair, and the total travel distance for all commuters in a SLA was calculated by summing all commuting trips that depart from a SLA. The average commuting distance for each SLA was finally derived from the total number of commuters in the SLA.

Figures 2 and 3 show that in both 1996 and 2006 the commuting distance tends to be shorter for workers who live closer to central city areas (e.g. Brisbane metropolitan area, and near the centres of the Gold Coast and Toowoomba City), whilst workers in the middle and outer suburbs and in rural locations tended to have longer, mainly cross-suburban commutes.

insert Figure 2 here

Figure 2. Average distance of JTW for years 1996

(note: numbers in brackets are the count of SLAs in a particular distance class)

insert Figure 3 here

Figure 3. Average distance of JTW for years 2006

(note: numbers in brackets are the count of SLAs in a particular distance class)

The result also reveals the difference in average commuting distance between 1996 and 2006 . A paired $t$ statistic was computed to test the significance in the change in the mean travel distance between 1996 and 2006. The result shows that the overall correlation between JTW distance in 1996 and 2006 was very high $(r=0.96)$, and the change in average JTW distance between $1996(15.75 \mathrm{~km})$ and $2006(15.95 \mathrm{~km})$ was not significant at regional scale ( $p$-value = 0.191). Figure 3 also reports that that there were numerous SLAs where residents travelled shorter distances to work (less than $10 \mathrm{~km}$ ) in 2006 but the number of SLAs where residents commuted $10 \mathrm{~km}$ to $30 \mathrm{~km}$ also marginally increased. The number of SLAs with their residents travelling longer distances (30 km or more) remained relatively stable between 1996 and 2006.

The change in average commuting distance between 1996 and 2006 is highlighted in Figure 4. The circular symbols represent the SLAs where the average commuting distance increased by more than their standard deviation (SD); and SLAs with the average commuting distance decreased by more than their SD are represented by square symbols. The result shows that over time a decrease in average commute distance occurred at the Port of Brisbane, along the

\footnotetext{
${ }^{2}$ The use of randomly distributed points can be affected by the size of a SLA. However, as the rural SLAs are often large geographical areas with more dispersed populations and employment activities, this approach is considered applicable.

${ }^{3}$ Comparing the Euclidian distance measure with that of network distance employed the former generally underestimated travel distance particularly in areas where the road network was sparse.
} 
Brisbane to Ipswich corridor, and for most Gold Coast suburbs. The possible reasons include fast urban growth and employment decentralization, which have introduced employment opportunities into these areas, especially at the Port of Brisbane. People living in or near these areas tend to have more local work opportunities and can commute relatively short distances. The areas with an increased commuting distance were highlighted as the central city areas (Brisbane CBD, coastal areas of Gold Coast city). The significant increase in reverse commuting from the inner city could be driven by decentralized employment towards outer urban areas. The increased commuting from inner city Brisbane can also be related to the increased commuting interactions between Brisbane and Gold Coast city. For example, there are increased number of professionals and government employees who live in the Brisbane City but commute to the Gold Coast. The result also indicates that the average commuting distance for outer suburbs and rural locations increased substantially compared with metropolitan suburbs, especially in some northern suburbs such as Caboolture and Esk and most suburbs around the city of Toowoomba. Tentatively, the increasing commute distances for outer suburbs can be partly explained by the new residents in such emerging peri-urban locations (e.g. Caboolture) who are still reliant on employment well outside their local area (e.g. Brisbane CBD).

\section{insert Figure 4 here}

Figure 4. Changes in average commuting distance between 1996 and 2006.

\subsection{JHB}

JHB refers to the spatial relationship between the number of jobs and housing units (or more accurately resident workers) within a given geographical area. Theoretically, if the number of jobs equals to the number of working residents in a geographical area, people will be able to work and live in the same area and long commutes can be avoided. The JHB has been long acknowledged as a planning solution to reduce travel demand (Cervero, 1989). In this study, we use jobs-housing ratio (JHR) (number of jobs per working resident) to measure the level of JHB.

JHR are often calculated solely for large administrative areas such as local governments (see Yates et al, 2006). The limitation of this approach is that it poorly accounts for the short commuting trips within a larger area, and the interactions between that area and surrounding locations. Furthermore, small areas, such as zones within the inner city, may have an internal JHR that suggests they have a mismatch, yet residents there may have very short commutes to nearby employment centres. In response, researchers have employed floating catchment areas to measure JHR (Peng, 1997; Wang, 2000; Luo, 2004). However, floating catchment areas in these studies are often defined by the buffered area of each travel zone and the catchment of households and jobs may be affected by the size and shape of the spatial unit.

In this study, we applied a modified approach to measure the JHR to overcome such spatial issues. First, a network distance buffer of $13.5 \mathrm{~km}$ was created for each zone (this being the median of the commuting distance for the whole study region). All jobs within $13.5 \mathrm{~km}$ travel on the road networks were regarded as 'local' to the SLA. The use of a median of commuting distance to measure JHR is supported in previous research as it reflects the outcome of the market forces that shape residential and job locations (Peng, 1997). The number of jobs and 
resident workers that lay within each buffer area was then summed, and the ratio of the total number of jobs to the total number of working residents was calculated for each SLA.

The resulting map of the JHR for 2006 is shown in Figure 5. The map presents a clear polycentric pattern that the higher JHR is concentrated nearer the Brisbane CBD, the cities of Gold Coast and Sunshine Coast and the regional centre of Toowoomba. The JHR for 1996 showed a very similar pattern to that presented for 2006, except for a higher JHR in the central urban areas. This means that the entire region was not significantly restructured over the period of 10 years, and most areas experienced both population and employment growth and therefore retained a similar JHR.

\section{insert Figure 5 here}

Figure 5. Distribution of the ratio of accessible jobs per resident for 2006

(within $13.5 \mathrm{~km}$ travel)

\section{Spatial effects of JHB on commuting distance}

The average commuting distance was plotted against the JHR for 1996 and 2006, respectively (see Figures 6 and 7). Both plots highlight that the majority of SLAs in the study region are job-housing imbalanced (e.g. JHR $<0.5$ or JHR $>1.5$ ) and that only a small number of areas ( $5 \%$ of total SLAs) have roughly an equal number of resident and employment (JHR $\approx 1$ ). The commuters living in the housing-rich and job-poor areas (e.g. JHR $<0.5 ; 15 \%$ of total SLAs) on average have a longer and more dispersed commuting pattern. The average commuting distance for housing-poor and job-rich areas (JHR > 1.5; 31\% of total SLAs) tends to be shorter and less dispersed. Therefore, at a regional level the average travel distance is negatively related to the JHR and the pattern of relationship is very consistent between 1996 and 2006.

insert Figure 6 here

Figure 6. Average commuting distance and JHR for SEQ SLAs in 1996.

\section{insert Figure 7 here}

Figure 7. Average commuting distance and JHR for SEQ SLAs in 2006.

Next, we plotted the changes in average commuting distance (at the SLA level) against the changes in JHR between 1996 and 2006 to explore the interaction between the two processes. Figure 8 shows that most SLAs are highly clustered within the centre of the graph suggesting no clear regional relationship between the change in commuting distance and the change in JHR. This relationship was expected to be negative, based on the hypothesis that people tend to travel shorter distances for work because of the increased local employment opportunity. 
Figure 8 also revealed that the changing JHR had a very different effect on average commuting distances in local areas.

insert Figure 8 here

Figure 8. Change in JHR and change in average commuting distance for SEQ SLAs between 1996 and 2006.

Because SEQ is a large geographical region, the relationship between change in JHR and commuting distance may present strong spatial variations. In this study, we focus on how the changes in JHR influence commuting behaviour across a large region. The specific locations where there is change at a local scale is a concern for planners. To identify local relationships between changes in JHR and commuting distance in SEQ over the past 10 years, a local exploratory spatial analysis method is applied. Local Indicators of Spatial Association (LISA) (Anselin, 1995) measures the tendency of each unit of the region (e.g. a SLA) to have a value that is correlated with the values in the nearby areas. In this research, LISA is for the first time, applied in a transport study to analyse whether changes in JHR cause changes in commuting distances at a local area scale, and the spatial distribution of these local relationships.

We plot the change in commuting distance against change in JHR, and use the four quadrants of the scatterplot to identify four types of relationships between two processes: High-High (HH), Low-Low (L-L), High-Low (H-L), and Low-High (L-H). For example, H-L relationship means a SLA with reduced commuting distance was surrounded by SLAs with increased JHR. The description of each of four types of spatial relationships is provided in Table 1.

Table 1

Description of 4 types of spatial relationship.

insert Table 1here

To assess the significance of the local relationships between change in JHR and commuting distance, local bivariate Moran's I statistics (Anselin, 1995) were used. The advantage of this local statistical method is that it not only measures local dependency within a single spatial unit (e.g. a SLA) but also provides a measure for the neighbourhood effects. It is used as a desirable method for detecting spatial arrangements of JHR in conjunction with commuting behaviour to investigate the existence of patterns of local dependence.

The local bivariate Moran's I can be defined as:

$$
I=Z_{i}^{d} \sum_{j} W_{i j} Z_{j}^{r}
$$

where $Z_{i}^{d}$ is the value of the change in commuting distance for the SLA $i$, and $Z_{j}^{r}$ is the change in JHR at neighbouring SLA $j$, it is among the identified neighbours of according to the spatial weight matrix $W_{i j}$.

Figure 9 shows the spatial distribution of the four types of local relationships between changes in JHR and commuting distance and the significance of the bi-variate local Moral's I statistics 
(represented by the $p$-value). It has been found that over time most areas with a strong increase or decrease in commuting distance had a strong association with the change in JHR in local areas, and that spatial relationship between the two processes varies greatly. Most $\mathrm{H}-\mathrm{L}$ relationships were clustered at the areas along the Brisbane-Ipswich corridor and inner suburban areas of Gold Coast City. The H-L relationships appeared to be more significant for Gold Coast suburbs ( $p$-value $=0.01$ ), indicating the decrease in commuting distance in those areas was strongly related to the increased JHR in the nearby areas. The very strong $\mathrm{H}-\mathrm{H}$ relationships ( $p$-value $=0.01$ ) were highly concentrated at the core Brisbane City area. This implies that despite the overall employment growth in the central Brisbane area, the increase in reverse commuting from the Brisbane CBD was significantly driven by decentralized employment at outer urban areas (not only at nearby inner urban areas). Such $\mathrm{H}-\mathrm{H}$ relationships were not observed in the Gold Coast CBD. Many outer suburbs and rural locations (e.g. suburbs around the City of Toowoomba) present significant L-H relationships ( $p$-value $=0.05$ ). This was possibly caused by decreased employment in agriculture sectors during the period, which forced the re-location of local workplaces to nearby town centres, and by the arrival of new residents who were trading off decreased house prices for longer commutes. The L-L effect occurred in some outer urban areas of Gold Coast City, implying that commuting patterns in those areas were less dependent on employment changes in surrounding areas. The decrease in the local commuting distance was more likely to be influenced by the change in local employment (within the SLA) than in the surrounding SLAs.

\section{insert Figure 9 here}

Figure 9. Spatial distribution of local correlation between change in JHR and change in average commuting distance (between 1996 and 2006)

In general, these findings support the commonly held views of much of the existing literature in the area that the co-location of employment and population does not necessarily have a significant effect on reducing average commuting distance (Wachs et al., 1993; Giuliano and Small, 1993; Peng, 1997; Wang, 2000). The change in commuting distance presents a very different relationship to the change in JHR at different local areas. Workers living in metropolitan areas showed both increases and decreases in commuting in response to urban growth and decentralization, whilst rural/regional workers tended to travel longer distances to work, due to economic restructuring and urbanization. The longer commuting travel may relate to the type of urban restructuring experienced in SEQ between 1996 and 2006. Though some employment decentralization has occurred, there were countervailing forces, with the Brisbane CBD also experiencing strong growth in white-collar employment during the period. The region has not seen strong clustering of employment into sub-regional centres, which have better access to the outer suburban/rural workers. Instead, new suburban employment was more laissez-faire and dispersed, reflecting weaknesses in planning, addressed in the more recent SEQ Regional Plan (Office of Urban Management, 2005).

Our results also indicate that despite policy settings promoting people's decisions on employment location, commuting choices appear to be affected by other factors. For example, it may be that there are major mis-matches in employment in specific SLAs or sub-regions according to the industry-type of employment and the skills and occupation of workers (socalled 'industry-occupation matching'). This could be resolved by investigating the relationships between commuting patterns in conjunction with social and spatial factors such as industry types, worker's skills and occupations, and household socio-economic status to derive a more in-depth understanding of the spatial and temporal variations in commuting. 


\section{Discussion and conclusion}

Understandings of temporal-spatial variation of JTW dynamics have gained prominence in transport studies given rising concerns about transport sustainability, congestion and greenhouse gas emissions. Previous studies on JTW dynamics tend to explore the relationship between commuting and urban structure based on limited geographical areas. This has called for more JTW research with other cities/regions to see if the methodologies and findings are transferable to a broader geographical context (i.e. Horner 2007). In this study, we suggest that such research should ideally be conducted for a large geographical region at a detailed spatial scale. Our work demonstrates value as tracking changes in commuting behaviour for large geographical areas is important for assessing structural changes in large regions and its broad impacts on transport. This paper has investigated change in JTW patterns based on disaggregated spatial analysis of JTW data over a decade (1996 - 2006), using SEQ as a case study area. SEQ is a fast-growing, large and poly-centric region with diverse geographic patterns of population and employment. The SEQ case demonstrates the viability of these methods and the usefulness of the innovative LISA statistical analysis, in particular, revealing impacts of land use change on commuting patterns. The methods advanced provides more insight into commuting dynamics, which will provide crucial information for land-use and transport planning and policy making to improve transport systems (e.g. excessive travel distance, fuel energy consumption, and congestion), and to ensure that urban and regional workers can equally benefit from regional growth and the planning and development of the region.

In terms of the SEQ case, we have found similar findings to what Horner (2007) found in Tallahasee, Florida, in that although SEQ has been relatively fast-growing (for an Australian city) a decade was insufficient to provide dramatic changes in commuting behaviour and JHR, other than at local scales. Through the application of local spatial statistical methods, the research revealed important differences in such local relationships between commuting and JHR in relation to different spatial locations and suggested possible reasons for such trends based on growth patterns of SEQ. These new findings complement existing works by not only exploring important variation of commuting change in a broader spatial context, but also presenting an approach for identifying the 'hot-spots' of commuting change that were highly related to the jobs-housing structure. This further advance on the methods previously advanced by Horner (2007) and Wang (2000) can help strategic planners spatially target different types of land use-transport interactions at specific areas and make relevant policy interventions.

While the techniques presented are applicable to model the spatial patterns of commuting over time, there remain a number of methodological concerns. Some of these have been discussed in previous research that drew attention to analytical problems faced when using spatially aggregated JTW data (i.e. see Horner, 2004). The major challenge is related to the modifiable areal unit problem (MAUP) (Openshaw 1977) which concedes that the scale and shape of large aggregate zones can affect the accuracy of results obtained from zone-based travel analysis (Horner and Murray, 2002). Although, this study tends to adopt improved GIS modelling techniques to assess JTW patterns, and it used mid-level spatial units in SLAs, the results were still not independent of the size and shape of these unit areas (as addressed in Section 4.2). There is a continuing research agenda to make refine and enhance such spatial and GIS techniques to resolve the geographical problems in transport research. A more advanced area interpolation technique is required to spatially integrate JTW matrices to better account for spatial heterogeneity of commuting flows within traffic zones (e.g., using an multi-class dasymetric method, see $\mathrm{Li}$ et al., 2007). In addition, a more spatial disaggregate method is needed to better measure individual travel patterns between aggregate trave zones, using travel 
survey data. Other avenues for further research include transferring these methods to use with more socially-disaggregated JTW analysis, to explore how JTW patterns differ according to industry sector, travel mode and gender, albeit this is reliant on access to data at reduced spatial scales (currently lacking in most jurisdictions, including SEQ), building on the work of Vandersmissen et al, (2003) and others. Merging these methods with studies using travel times on the road network, and access via the public transport network, may also provide a deeper and more robust understanding of the geographical and temporal variations of JTW across a region.

\section{References}

Anselin, L., 1995. Local indicators of spatial association - LISA. Geographical Analysis 27(2), 93-115.

Cervero, R., 1989. Jobs-Housing Balancing and Regional Mobility. Journal of the American Planning Association 55(2), 136-150.

Cervero, R., Wu, K.L., 1998. Sub-Centring and Commuting: evidence from the San Francisco Bay Area, 1980-90. Urban Studies 35(7), 1059-1076.

Christopher, E., Matthew, R., Siim S., 1995. Changes in the directions of urban travel for the Chicago area 1970 to 1990. Transportation Research Record 1477, 48-57.

Gipps, P., Brotchie, J., Hensher, D., Newton, P., O’Connor, K., 1997. Journey to Work: Employment and the Changing Structure of Australian Cities. Research Monograph 3, Australian Housing and Urban Research Institute, Melbourne.

Giuliano, G., Small K.A., 1993. Is the journey to work explained by urban structure? Urban studies 30(9), 1485-1500.

Horner, M.W., Murray, A.T., 2003. A multi-objective approach to improving regional jobshousing balance. Regional Studies 37(2), 135-146.

Horner, M.W., 2004. Spatial dimensions of urban commuting: A review of major issues and their implications for future geographical research. The Professional Geographer 56(2), 16073.

Horner M.W., 2007. A multi-scale analysis of urban form and commuting change in a small metropolitan area (1990-2000). Annals of Regional Science 41, 315-332;

Langford, M., 2006. Obtaining population estimations in non-census reporting zones: An evaluation of the 3-class dasymetric method. Computers, Environment and Urban Systems 30, 161-180.

Li, T, Pullar, D, Corcoran, J \& Stimson, R., 2007. A comparison of spatial disaggregation techniques as applied to population estimation for South East Queensland (SEQ), Applied GIS, 3(9), 1-16. 
Luo, W., 2004. Using a GIS-based floating catchment method to assess areas with shortage of physicians. Health and Place, 10, 1-11.

Mees, P.G., O’Connell, K., Stone, J., 2008. Travel to Work in Australian Capital Cities, 19762006. Urban Policy and Research 26(3), 363-378.

Mogridge, M.H., 1979. Changing spatial patterns in the journey-to-work: A comparison of the 1966 and 1971 census data in London. Urban Studies 16, 179-190.

Nielsen, T., Hovgesen, H., 2008. Exploratory mapping of commuter flows in England and Wales. Journal of Transport Geography 16(2), 167-256.

O'Connor, K., 1978. The journey to work of inner city residents in Melbourne 1966 and 1971. Australian Geographical Studies 16, 73-81.

O'Connor, K., 1980. The analysis of journey to work patterns in human geography. Progress in Human Geography 4(4), 475-499.

O’Kelly, M.E. Lee, W., 2005. Disaggregate journey-to-work data: Implications for excess commuting and jobs housing balance. Environment and Planning A 37, 2233-2252.

Office of Urban Management., 2005. South East Queensland Regional Plan 2005-2006. Series, Office of Urban Management, Brisbane, Australia.

Office of Economic and Statistical Research., 2006. Journey to Work: South East Queensland, Census 2006 Bulletin 11, Office of Economic and Statistical Research, Brisbane, Australia.

Openshaw, S., 1977. A geographical solution to scale and aggregation problem in region building, partition and spatial modelling. Transaction of the Institute of British Geographers, New Series 2, 459-472.

Peng, Z., 1997. The jobs-housing balance and urban commuting. Urban Studies 34, 1215-1235.

Rickwood, P., Glazebrook, G., 2007. Urban Structure and Commuting in Australian Cities. Paper presented at State of Australian Cities Conference, Adelaide, December 2007

Spearritt, P., 2009. The 200 km city: Brisbane, the Gold Coast and Sunshine Coast. Australian Economic History Review 49, 87-106.

Sakanishi, A., 2006. Commuting patterns in the Osaka metropolitan area: A GIS-based analysis of commuter rail passengers. RURDS 18(1), 41-59.

Sultana, S., 2002. Job/housing imbalance and commuting time in the Atlanta metropolitan area: exploration of causes of longer commuting time. Urban Geography 23(8): 728-749.

Weber, J., Sultana, S., 2007. Journey-to-work patterns in the age of sprawl: Evidence from two midsize southern metropolitan areas. The Professional Geographer 59(2), 193-208.

Titheridge, H., Hall, P., 2006. Changing travel to work patterns in South East England. Journal of Transport Geography 14, 60-75. 
Vandersmissen, M.H., Villeneuve, P., Theriault, M., 2003. Analyzing changes in urban form and commuting time. The Professional Geographer 55(4), 446-463.

Wachs, M., Taylor, B.D., Levine, N, Ong, P., 1993. The changing commute: A case study of the jobs-housing relationship over time. Urban Studies 30(10), 1711-1729.

Wang, F., 2000. Modelling commuter patterns in Chicago in a GIS environment: A job accessibility perspective. The Professional Geographer 52(1), 120-133.

Yates, J., B. Randolph, and D. Holloway. 2006. Housing affordability, occupation and location in Australian cities and regions, AHURI Final Report No. 91. Melbourne, Australia: Australian Housing and Urban Research Institute.

\section{Appendix}

List of Figures

Figure 1. Study area

Figure 2. Average distance of JTW for years 1996

Figure 3. Average distance of JTW for years 2006

Figure 4. Changes in average commuting distance between 1996 and 2006.

Figure 5. Distribution of the ratio of accessible jobs per resident for 2006

Figure 6. Average commuting distance and JHR for SEQ SLAs in 1996.

Figure 7. Average commuting distance and JHR for SEQ SLAs in 2006.

Figure 8. Change in JHR and change in average commuting distance for SEQ SLAs between 1996 and 2006

Figure 9. Spatial distribution of local correlation between change in JHR and change in average commuting distance (between 1996 and 2006)

\section{List of Tables}

Table 1. Description of 4 types of spatial relationship. 